\title{
Affinity Entrapment of Oligosaccharides and Glycopeptides Using Free Lectin Solution
}

\author{
Masahiro YodoshI, Takehiro OYaMa, Ken MASAKI, Kazuaki KAKEHI, Takao HaYAKaWA, and \\ Shigeo SUZUKI ${ }^{\dagger}$
}

Faculty of Pharmaceutical Sciences, Kinki University, 3-4-1 Kowakae, Higashi-osaka, Osaka 577-8502, Japan

\begin{abstract}
Two procedures were proposed for the specific recovery of fluorescent derivatives of glycoprotein-derived oligosaccharides and tryptic glycopeptides using certain plant lectins. The first was based on the salting out of oligosaccharide-lectin conjugates with ammonium sulfate. Oligosaccharides specifically bound to lectins were recovered free from lectins using ethanol precipitation after dissolution in water. This method enabled group separation of 2-aminopyridine-labeled oligosaccharides derived from ovalbumin to galacto-oligosaccharides and agalacto-oligosaccharides by Ricinus communis agglutinin, and to high mannose- and hybrid-type oligosaccharides by wheat-germ agglutinin. Fractional precipitation based on differences in affinity for concanavalin A was accomplished by adding an appropriate concentration of methyl $\alpha$-mannoside as an inhibitor. In the second method, tryptic digests of glycoproteins were mixed with a lectin solution, and the glycopeptide-lectin conjugates were specifically trapped on a centrifugal ultrafiltration membrane with cut-off of $10 \mathrm{kD}$. Trapped glycopeptides, as retentates, were passed through membranes by resuspension in diluted acid. This method is particularly useful for the enrichment of glycopeptides in protease digestion mixtures for glycosylation analyses by liquid chromatography-mass spectrometry.
\end{abstract}

(Received January 20, 2011; Accepted March 4, 2011; Published April 10, 2011)

\section{Introduction}

Lectins are proteins of non-immune origin that interact specifically with carbohydrates without modifying them. ${ }^{1,2}$ Several reports have stated that plant lectins bind primarily to monosaccharides, ${ }^{3}$ but that they bind to specific sequences of oligosaccharides with higher affinity. For example, concanavalin A (Con A) binds to $\alpha$-glucoside and $\alpha$-mannoside with a $K_{\text {a }}$ on the order of $10^{3}$, but a $K_{\mathrm{a}}$ of $10^{6}$ to $10^{7}$ engenders binding to high mannose-type oligosaccharides. ${ }^{4,5}$ In addition, the binding capability of lectins might not reflect the structural differences of the oligosaccharides. Therefore, the inhibitory action is dependent only on the concentration of inhibitors and their affinity for the lectins. High specificity of plant lectins against certain oligosaccharides is helpful for profiling in glycoproteomic analyses. Lectin affinity capillary electrophoresis ${ }^{6,7}$ and lectin microarray ${ }^{8}$ have been proposed for this purpose. Moreover, the combinational use of affinity columns of immobilized lectins is useful in sorting complex mixtures of oligosaccharides based on structural differences. ${ }^{9}$ In a typical separation procedure, the bound oligosaccharides are eluted from the lectin affinity columns by the addition of haptenic sugars. ${ }^{10}$ More recently, the use of high-performance lectin-affinity enrichment of glycoprotein using a series of silica-bound lectins at microscale levels has been reported. ${ }^{5,11-13}$ This enrichment procedure was combined with nano-liquid chromatography-tandem mass spectrometry (nano-LC-MS) to identify numerous glycoproteins. ${ }^{13}$ A multi-lectin column prepared by mixing

$\dagger$ To whom correspondence should be addressed.

E-mail: suzuki@phar.kindai.ac.jp three lectin-immobilized gels was successively used in enrichment of serum glycoproteins. ${ }^{14}$ However, despite the high efficiency of the combinational use of lectin-affinity columns, few studies have used that method because it necessitates special care for the preparation and maintenance of affinity columns. Recently, Hong et al. proposed ultrafiltration as a convenient tool to trap an affinity complex instead of using affinity columns, and they used the method to identify $\mathrm{Cu}^{2+}$-protein adducts in saliva. ${ }^{15}$

We report on simple methods for the specific recovery of glycoconjugates from complex samples using lectins without immobilization to a support. Because of the strong affinity of lectins to glycoprotein-derived oligosaccharides, the oligosaccharide-lectin complex is highly stable and not dissociated by precipitation using ammonium sulfate. The precipitates were resuspended in water; oligosaccharides specifically bound to a lectin were dissociated by alcoholic denaturation. This method was applied to the specific fractionation of 2-aminopyridine (AP) derivatives of ovalbumin-derived oligosaccharides. Another method was developed for glycopeptide enrichment based on the specific recovery of lectin-oligosaccharide complexes on a centrifugal ultrafiltration membrane with a cut-off of $10 \mathrm{kDa}$. Glycopeptides specifically trapped on a membrane were recovered by treatment with diluted acid. This method simplified the interpretation of LC/MS data for glycopeptide analyses.

Abbreviations: AP, 2-aminopyridine; WGA, wheat germ agglutinin; $\mathrm{RCA}_{120}$, Ricinus communis agglutinin; Con A, concanavalin A; SSA, Sambucus sieboldiana lectin; MAM, Maackia amurensis agglutinin; Gal, D-galactose; GlcNAc, $N$-acetyl D-glucosamine; Man, D-mannose; Fuc, L-fucose; NeuAc, $N$-acetyl neuraminic acid. 


\section{Experimental}

\section{Chemical and buffers}

The lectins used in the present study, Con A, wheat germ agglutinin (WGA), Ricinus communis agglutinin ( $\left.\mathrm{RCA}_{120}\right)$, Sambucus sieboldiana lectin (SSA), and Maackia amurensis agglutinin (MAM), were purchased from Seikagaku Corp. (Tokyo, Japan). Ovalbumin was prepared according to a procedure described by Kekwick and Cannan. ${ }^{16}$ L-1-Tosylamido2-phenylethyl chloromethyl ketone (TPCK)-treated trypsin from bovine pancreas (EC 3.4.21.4), bovine pancreas ribonuclease B, human transferrin, and iodoacetamide were obtained from Sigma-Aldrich, Japan K.K. (Tokyo, Japan). Methyl and $p$-nitrophenyl $\alpha$-D-mannopyranoside, and AP were from Nacalai Tesque Incorporated (Karasuma, Kyoto, Japan). Before use, AP was recrystallized thrice from hexane. AP-labeled oligosaccharides were prepared from ovalbumin, as described previously. ${ }^{17} \quad$ Peptide- $N^{4}$-(acetyl- $\beta$-glucosaminyl)asparagine amidase (PNGase F, EC 3.2.2.18) was obtained from F. Hoffman La Roche Ltd. (Tokyo, Japan). An ultrafiltration membrane (Ultrafree-MC-10) was obtained from Nihon Millipore Ltd. (Tokyo, Japan). Other reagents and solvents were of the highest grade commercially available.

\section{Procedures for specific recovery}

Entrapping methods were optimized based on the affinity between $p$-nitrophenyl $\alpha$-mannoside and Con A. The amounts bound to Con A were determined using capillary electrophoresis with $\mathrm{HP}{ }^{3 \mathrm{D}} \mathrm{CE}^{\circledR}$ equipment (Agilent Technologies, Santa Clara, USA) using a fused-silica capillary $(50 \mu \mathrm{m}$ i.d., $60 \mathrm{~cm})$ and $100 \mathrm{mM}$ sodium borate buffer $(\mathrm{pH} 10.0)$ as the background electrolyte.

Method A: Saccharide samples $(\approx 2 \mathrm{nmol}$, or oligosaccharides derived from $2 \mu \mathrm{g}$ of ovalbumin) were dissolved in $10 \mu \mathrm{L}$ of $50 \mathrm{mM}$ Tris- $\mathrm{HCl}$ (pH 7.0) containing $150 \mathrm{mM} \mathrm{NaCl}, 1 \mathrm{mM}$ $\mathrm{CaCl}_{2}$ and $1 \mathrm{mM} \mathrm{MgCl}$ (TBS) and mixed with a lectin solution $(0.5 \mathrm{mg}$ in $20 \mu \mathrm{L}$ of TBS) in an ice bath. The solution was allowed to stand for $2 \mathrm{~min}$, and was then mixed with $100 \mu \mathrm{L}$ of a saturated aqueous solution of ammonium sulfate. The suspended solution was centrifuged at $5000 \mathrm{rpm}$ for $2 \mathrm{~min}$ at $4{ }^{\circ} \mathrm{C}$. The supernatant was removed; the precipitate was washed by resuspension in saturated ammonium sulfate, and then centrifuged at $5000 \mathrm{rpm}$ for $2 \mathrm{~min}$ at $4^{\circ} \mathrm{C}$, with removal of the supernatant in the same manner. Precipitated residues were dissolved in $10 \mu \mathrm{L}$ of water. Lectin was then removed from the reaction mixture by centrifugation at $10000 \mathrm{rpm}$ for $2 \mathrm{~min}$ at $4{ }^{\circ} \mathrm{C}$, followed by mixing with $40 \mu \mathrm{L}$ of ethanol. The supernatant was evaporated to dryness and stored at $4{ }^{\circ} \mathrm{C}$ until use.

Method B: The sample solution $(5 \mu \mathrm{g}$ dissolved in $10 \mu \mathrm{L}$ of TBS) was mixed with a lectin solution $(0.5 \mathrm{mg}$ in $20 \mu \mathrm{L}$ of TBS) in an ice bath and allowed to stand for $2 \mathrm{~min}$. The lectin complexation solution was poured onto a centrifugal ultrafiltration tube $(10 \mathrm{kDa})$. The solution that had passed through was discarded after centrifugation at $10000 \mathrm{rpm}$ for $10 \mathrm{~min}$. The retentate was mixed with $100 \mu \mathrm{L}$ of $10 \mathrm{mM} \mathrm{HCl}$. Glycoconjugates dissociated from a lectin were recovered as a filtration solution by centrifugation ( $10000 \mathrm{rpm}$ for $10 \mathrm{~min}$ ). The obtained solution was dried and stored at $4{ }^{\circ} \mathrm{C}$.

\section{Analyses of AP-labeled ovalbumin-derived oligosaccharides}

The AP-labeled oligosaccharides prepared from $50 \mu \mathrm{g}$ of ovalbumin were dissolved in $500 \mu \mathrm{L}$ of water. A $20-\mu \mathrm{L}$ portion was separated using high-performance liquid chromatography (HPLC) with a gradient HPLC system built of two 870 pumps and an FP-2020plus fluorescence monitor with a flow cell with a volume of $10 \mu \mathrm{L}$ (Jasco Incorporation, Tokyo, Japan). Data were collected by SmartChrom software (KYA Technologies Corporation, Tokyo, Japan). Labeled oligosaccharides were separated on a reversed-phase column $\left(\mathrm{C}_{18}, 5 \mu \mathrm{m}, 6 \mathrm{~mm}\right.$ i.d., $15 \mathrm{~cm}$; Nacalai Tesque Incorporation, Kyoto, Japan) with a linear gradient of $0.1-0.425 \%$-butanol in $100 \mathrm{mM}$ triethylammonium acetate $(\mathrm{pH} 4.0)$ for $100 \mathrm{~min}$ at a flow rate of $1.0 \mathrm{~mL} / \mathrm{min}$. Fluorometric detection of the analytes was accomplished at an excitation wavelength of $321 \mathrm{~nm}$ and an emission wavelength of $383 \mathrm{~nm}$.

\section{LC-MS analyses of tryptic glycopeptides}

Tryptic digestion was carried out according to the manufacturer's protocol. Liquid chromatography/electrospray ionization-mass spectrometry (LC/ESI-MS) analyses were conducted using ultra-performance liquid chromatography (UPLC; Prominence series; Shimadzu Corp., Kyoto, Japan) coupled to quadruple ion trap-time of flight (QIT-TOF) equipment (Shimadzu Corp.). Tryptic digests were separated on a reversed-phase column $(15 \mathrm{~cm} \times 2.1 \mathrm{~mm}$ i.d., HiQsil C18-3; KYA Technologies Corp.) at a flow rate of $0.15 \mathrm{~mL} / \mathrm{min}$ using gradient elution. The gradient program was $0.1 \%$ trifluoroacetic acid (TFA; eluent $\mathrm{A}$ ) and $60 \%(\mathrm{v} / \mathrm{v})$ acetonitrile in $0.1 \%$ TFA (eluent B); $5-5 \% \mathrm{~B}$ at $0-2 \mathrm{~min}, 5-60 \% \mathrm{~B}$ at $2-42 \mathrm{~min}$, and $60-95 \% \mathrm{~B}$ at $42-52 \mathrm{~min}$. The eluate from the column was introduced to an orthogonal electrospray interface of a QIT-TOF mass spectrometer with argon gas for ion cooling and collision-induced dissociation (CID) experiments. The QIT-TOF-MS was operated in the positive-ion mode with the probe voltage set to $4.5 \mathrm{kV}$, a detector voltage of $-1.7 \mathrm{kV}$, a capillary temperature of $200^{\circ} \mathrm{C}$, and a nebulizer gas flow of $1.5 \mathrm{~L} / \mathrm{min}$. Mass spectra were acquired over the scan range of $\mathrm{m} / \mathrm{z} 500-2000$ with a scan rate of $5 \mathrm{~s} / \mathrm{scan}$. The obtained extracted ion chromatograph (EIC) and MS data were processed using LCMSsolution software (Shimadzu Corp.).

\section{Results and Discussion}

We proposed two procedures for the specific extraction of glycoconjugates (Fig. 1). In method A, a lectin solution was mixed with a saccharide sample. Lectin-saccharide complexes were salted out by adding ammonium sulfate. The precipitate was dissolved in water, and trapped saccharides freed from lectins by adding ethanol. In method $\mathrm{B}$, a lectin solution was mixed with a saccharide sample, and the mixture passed through an ultrafiltration membrane for a cut-off of $10 \mathrm{kDa}$ by centrifugation. Substances unbound to the lectin were removed in this process. Saccharides residing as lectin complexes on the ultrafiltration membrane were recovered as filtrate by centrifugation after mixing the retentates with acid solution.

Method A was optimized by tuning two factors: (i) the amounts or molar ratio of the saccharide specimen and the lectin in the reaction solution; and (ii) the volume of a saturated solution of ammonium sulfate added to precipitate the complex of the lectin with an oligosaccharide. We chose $p$-nitrophenyl $\alpha$-mannoside and Con A as model systems to optimize the factors. Con A is most commonly used for the affinity chromatography of oligosaccharides; the $K_{\mathrm{a}}$ value for $p$-nitrophenyl $\alpha$-mannoside is reportedly $7200 \mathrm{M}^{-1}$. $\quad$ Results showed that a sample containing $<2 \mathrm{nmol}$ of saccharides was quantitatively recovered by adding $0.5 \mathrm{mg}$ of lectin and $100 \mu \mathrm{L}$ of a saturated ammonium sulfate solution.

In method B, the recovery of mannoside was dependent only 
Method A

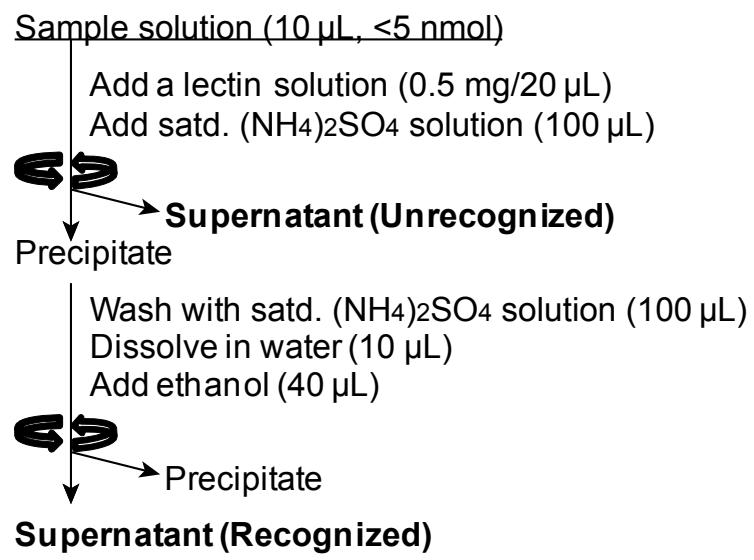

\section{Method B}

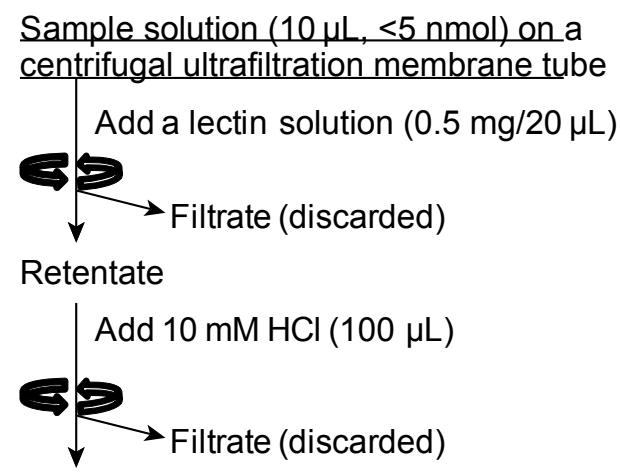

Filtrate as free saccharides

Fig. 1 Procedure for the fractionation of glycans using ammonium sulfate fractionation (method A) and a centrifugal ultrafiltration membrane (method B). TBS: $50 \mathrm{mM}$ Tris- $\mathrm{HCl}(\mathrm{pH} 7.0)$ containing $150 \mathrm{mM}$ sodium chloride, $1 \mathrm{mM} \mathrm{CaCl} 2$ and $1 \mathrm{mM} \mathrm{MgCl}$. Lectin was dissolved in TBS to a concentration of $25 \mathrm{mg} / \mathrm{mL}$.

upon its molar ratio to Con A. When $0.5 \mathrm{mg}$ of Con A dissolved in $20 \mu \mathrm{L}$ of buffer was mixed with $10 \mu \mathrm{L}$ of $p$-nitrophenyl $\alpha$-mannoside at various concentrations, the recovery decreased linearly, concomitantly with the increase in the mannoside concentration. The recovery ratio changed from $>95 \%$ for $0.2 \mathrm{nmol}$ of mannoside to $45 \%$ for $5 \mathrm{nmol}$ of mannoside. The recovery ratio of $90 \%$ obtained using $1 \mathrm{nmol}$ of mannoside seemed to be sufficient for trapping glycoprotein-derived oligosaccharides because their affinity should exceed $10^{5} \mathrm{M}^{-1}$.

Application of method A for specific extraction of ovalbumin-derived oligosaccharides

Method A was applied to the specific recovery of AP-labeled oligosaccharides derived from ovalbumin. The oligosaccharide structures are presented in Table 1. This glycoprotein contains three high mannose-type, one complex-type, and five bisected hybrid-type oligosaccharides, two of which contain one Gal residue. The AP derivatives of these oligosaccharides indicated good resolution in reversed-phase HPLC, which could resolve all except one pair of oligosaccharides $\left(G_{1}\right.$ and $\left.G_{2}\right)$ (bottom trace of Fig. 2). ${ }^{18}$ We examined method A for the fractionation and recovery of these oligosaccharides using three lectins: $\mathrm{RCA}_{120}$, WGA and Con A.

Figure 2 shows the results of fractions obtained using $\mathrm{RCA}_{120}$ and WGA lectins. Ammonium sulfate precipitates as $\mathrm{RCA}_{120}$ complexes comprised two components corresponding to peaks $\mathrm{G}_{1}$ and I (Fig. 2(a)), both of which contained Gal. The supernatant included the remainder of the oligosaccharides (peaks A-F, and H) (Fig. 2(b)). A small peak assignable to $G$ in the supernatant indicated the presence of the Gal-free oligosaccharide $\mathrm{G}_{2}$. Consequently, $\mathrm{RCA}_{120}$ showed an affinity for the Gal-containing hybrid-type oligosaccharide $\left(\mathrm{G}_{1}\right.$ and $\left.\mathrm{I}\right)$. The same strategy was also used on the GlcNAc-specific lectin WGA. As depicted in Figs. 2(c) and (d), peaks D - I were inferred to indicate WGA precipitates; peaks A - C corresponding to high mannose-type oligosaccharides were not recognized by WGA, and were recovered as a supernatant fraction. In this chromatogram, some of the recovered peaks indicated apparently reduced intensities, which may be due to a loss in the step of denaturation with ethanol (i.e. unspecific coprecipitation with lectin). All $N$-linked oligosaccharides contained a chitobiose
Table 1 Oligosaccharide derived from ovalbumin and binding specificities to lectins

\begin{tabular}{|c|c|c|c|c|}
\hline \multirow{2}{*}{ Peak No. } & \multirow{2}{*}{ Structure } & \multicolumn{3}{|c|}{ Affinity to } \\
\hline & & $\mathrm{RCA}_{120}$ & WGA & Con A \\
\hline A & & - & - & ++ \\
\hline B & & - & - & ++ \\
\hline $\mathrm{C}$ & & - & - & ++ \\
\hline D & & - & + & ++ \\
\hline $\mathrm{E}$ & & - & + & + \\
\hline $\mathrm{F}$ & & - & + & ++ \\
\hline $\mathrm{G}_{1}$ & & + & + & ++ \\
\hline $\mathrm{G}_{2}$ & & - & + & + \\
\hline $\mathrm{H}$ & ofo & - & + & \pm \\
\hline I & 象ono & + & + & \pm \\
\hline
\end{tabular}

core (GlcNAc $\beta 1-4 \mathrm{GlcNAc}$ ) in their reducing termini, but terminal GlcNAc residues were transformed to linear aminoalditol derivatives by labeling with AP (which impaired their binding capability to WGA). These results indicated that WGA specifically recognized bisecting GlcNAc residues commonly existing in these hybrid-type oligosaccharides. These results showed that $\mathrm{RCA}_{120}$ and WGA recognized oligosaccharides having terminal $\mathrm{Gal}$ and GlcNAc residues, respectively. Both lectins, therefore, have direct usefulness for the discrimination of hybrid-type oligosaccharides. The results 


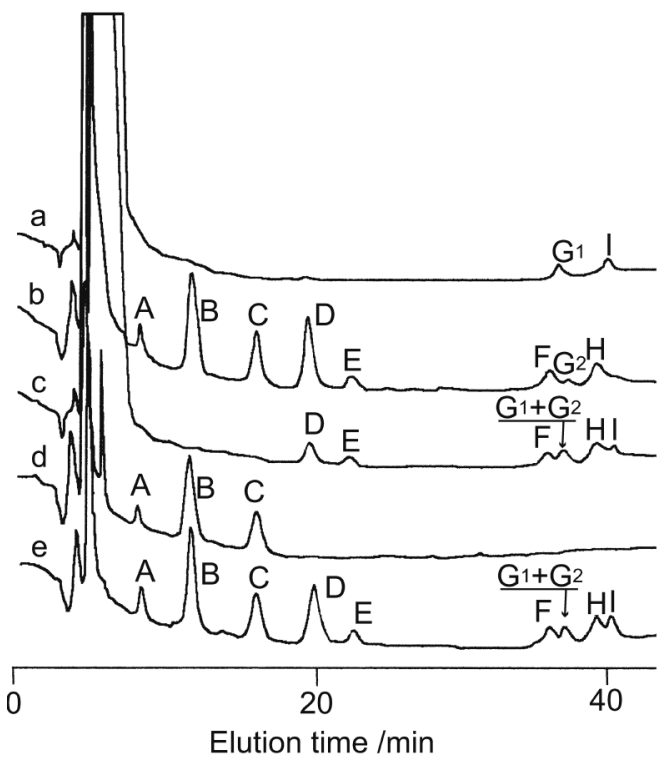

Fig. 2 Analysis of AP-labeled glycans from ovalbumin (e), and their fractions, as retained and unretained as $\operatorname{RCA}_{120}$ complex $(\mathrm{a}, \mathrm{b})$ and those as WGA complex (c, d). Analytical conditions: Cosmosil C18 $(15 \mathrm{~cm} \times 6 \mathrm{~mm}$ i.d. $)$ column, $1.0 \mathrm{ml} / \mathrm{min}$ flow rate, $100 \mathrm{mM}$ triethylamine/acetic acid ( $\mathrm{pH} 4.0$ ) with a linear gradient of $n$-buthanol $(0.1-0.425 \%$ over $100 \mathrm{~min})$ used as the eluent. Peak identification is presented in Table 1.

underscored the usefulness of the free-lectins strategy for the specific recovery of oligosaccharides with high recovery.

In contrast to WGA and $\mathrm{RCA}_{120}$, Con $\mathrm{A}$ seemed to have affinity for all oligosaccharides derived from ovalbumin, and all saccharides were recovered as a Con A complex (Fig. 3). Con $\mathrm{A}$ is known to recognize oligosaccharides containing two mannose residues having free hydroxyl groups at their C-3, C-4, and C-6 positions. Actually, Con A shows weak affinity for biantennary oligosaccharides. The interaction between Con A and high mannose-type oligosaccharides can be classified into two groups. Affinity for high mannose-type oligosaccharides containing $>7$ mannose residues indicates strong binding $\left(K_{\mathrm{a}}\right.$ $>10^{7} \mathrm{M}^{-1}$ ); these binding constants are one order of magnitude higher than that of high mannose-type oligosaccharides containing $<6$ mannose residues $\left(K_{\mathrm{a}} \approx 10^{6} \mathrm{M}^{-1}\right){ }^{4}$ We evaluated the specific recovery of a series of high mannose-type and hybrid-type oligosaccharides based on the difference of affinity for Con A by precipitating the complex of AP-oligosaccharides and Con A in the presence of methyl $\alpha$-mannoside of various concentrations (Fig. 3). The binding constant of methyl $\alpha$-mannoside for Con A has been reported to be $7200 \mathrm{M}^{-1,5}$ which is lower than that of biantennary complex-type oligosaccharides. All ovalbumin oligosaccharides were recognized in the absence of mannoside. With increasing concentrations of methyl $\alpha$-mannoside, some peaks disappeared; peaks $\mathrm{H}$ and I disappeared upon the addition of $10 \mathrm{mM}$ methyl $\alpha$-mannoside, and peak $\mathrm{E}$ disappeared upon the addition of $25 \mathrm{mM}$ of methyl $\alpha$-mannoside, respectively. However, other peaks were trapped by $\mathrm{Con} A$ in the presence of $50 \mathrm{mM}$ mannoside.

Though relational plots in Fig. 3 may include some uncertainty, because some peaks indicated more than $100 \%$ of recovery, the inhibitory assay using methyl $\alpha$-mannoside to Con A seems to be useful in estimating their binding strength of oligosaccharides. The peak area or retention behaviors in affinity chromatography

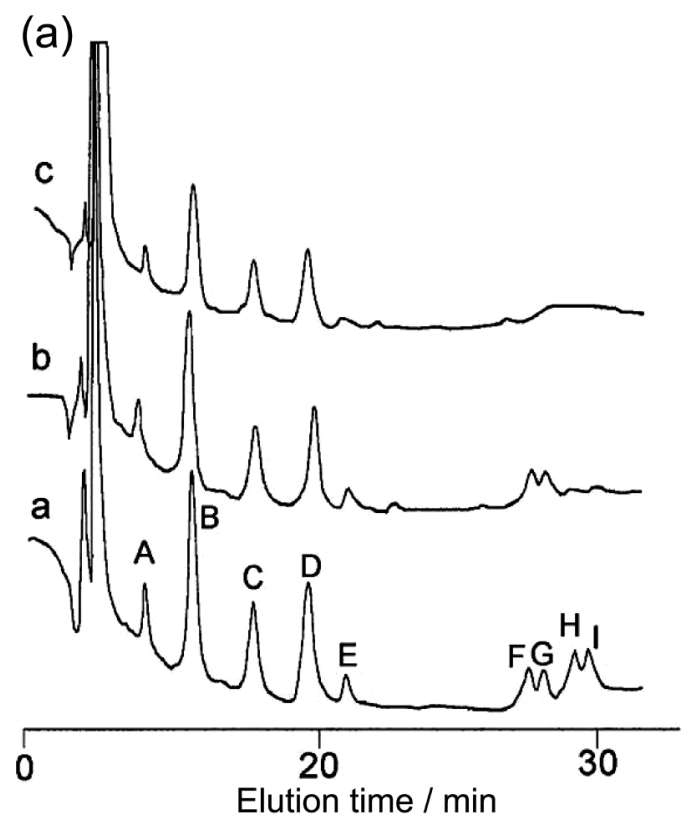

(b)

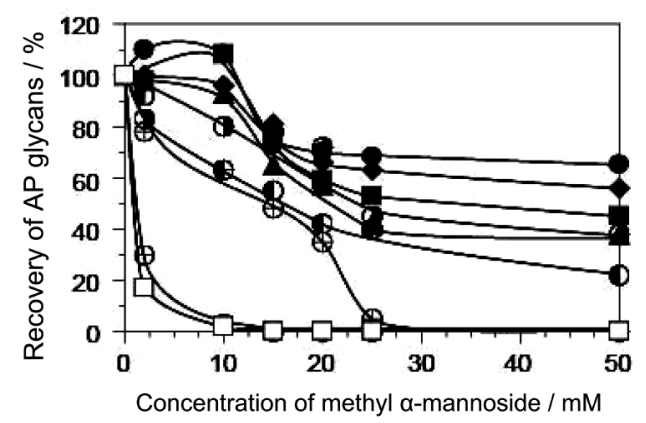

Fig. 3 Analysis of AP-labeled glycans from ovalbumin recovered as Con A complex in the presence of $0 \mathrm{mM}$ (a), $20 \mathrm{mM}$ (b), and $50 \mathrm{mM}$ (c) of methyl $\alpha$-mannoside (a) and effect of the recoveries of AP-labeled glycans under the presence of methyl $\alpha$-mannoside (b). The peak assignment and analytical conditions are identical to those in

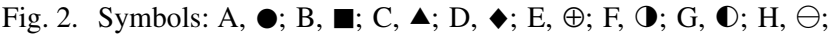
I, $\square$.

correlate directly to the binding constants. However, the binding plots of these oligosaccharide to Con A showed sigmoid curves, which make it impossible to determine binding constants. This is attributable to the large difference in the binding constants of Con A to mannose $\left(10^{3} K_{\mathrm{a}}\right)$ and glycoprotein-derived oligosaccharides $\left(\mathrm{ca} .10^{6} K_{\mathrm{a}}\right)$.

\section{Application of method $B$ for the enrichment of tryptic glycopeptides}

Method B was applied to LC-MS analyses of glycopeptides in digestion mixtures. Ribonuclease $\mathrm{B}$ from bovine pancreas contains a series of high mannose-type oligosaccharides at a single glycosylation site at $\mathrm{Asn}^{60} .{ }^{19}$ Trypsin predominantly cleaves peptide chains at the carboxyl side of the amino acids of lysine or arginine. Therefore, this glycoprotein is estimated to be digested to $>15$ species of peptides. Tryptic digestion indicated numerous peaks in the total ion current (TIC) spectra obtained on reversed-phase LC/MS in the positive ion mode (Fig. 4(a)). The sample was treated with Con A, and the retentates recovered and analyzed under the conditions depicted 


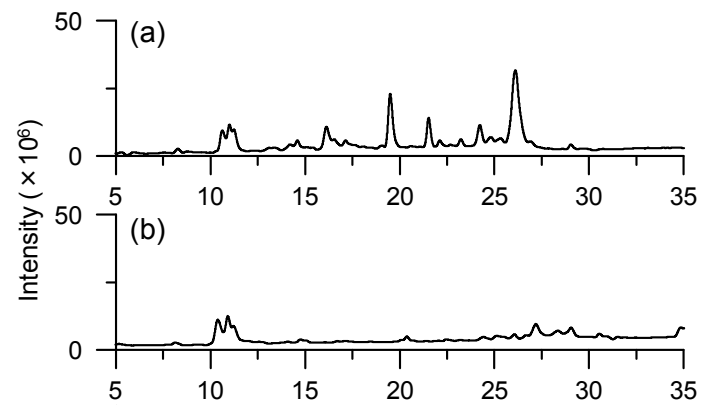

(c)

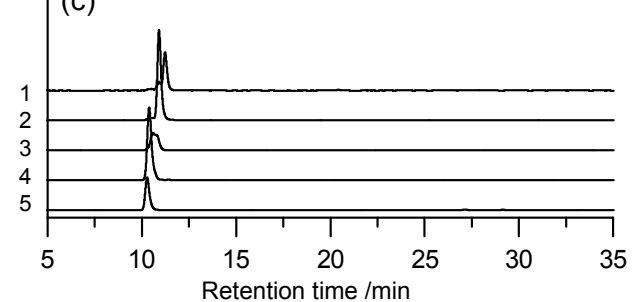

\begin{tabular}{ccccl}
\hline No. & $\begin{array}{c}\text { measured } \\
m / z\end{array}$ & $z$ & $\begin{array}{c}\text { glycosylation } \\
\text { site }\end{array}$ & $\begin{array}{c}\text { glycan structure with identification } \\
\text { based on } m / z\end{array}$ \\
\hline 1 & 845.86 & 2 & & {$[\mathrm{Hex}]_{5}[\mathrm{HexNAc}]_{2}$} \\
2 & 926.89 & 2 & & {$[\mathrm{Hex}]_{6}[\mathrm{HexNAc}]_{2}$} \\
3 & 1079.92 & 2 & $\mathrm{Asn}^{60}$ & {$[\mathrm{Hex}]_{7}[\mathrm{HexNAc}]_{2}$} \\
4 & 1089.94 & 2 & & {$[\mathrm{Hex}]_{8}[\mathrm{HexNAc}]_{2}$} \\
5 & 1169.97 & 2 & & {$[\mathrm{Hex}]_{9}[\mathrm{HexNAc}]_{2}$} \\
\hline
\end{tabular}

Fig. 4 Total ion current chromatograms of reversed-phase LC/ESI-MS analysis of glycopeptides derived from bovine ribonuclease B digested with trypsin before (a) and after (b) specific extraction with Con A. Extracted ion chromatograms (c) correspond to trivalent molecular ions of glycopeptides with specific sequences listed in the table. Analytical conditions: column, HiQsil C18-3 ( $15 \mathrm{~cm} \times 2.1 \mathrm{~mm}$ i.d.); flow rate, $0.15 \mathrm{~mL} / \mathrm{min}$; eluent, water-acetonitrile with the gradient program described in the Experimental section.

in Fig. 4(b). Intense peaks were observed at $10-12$ min. The extracted ion chromatogram (EIC) of each peak clearly indicated that these peaks corresponded to glycopeptides with a specific length of high mannose-type oligosaccharides with a common peptide sequence of NLTK. Their elution order matched that of the decrease in mannose residues. Recoveries of these glycopeptides were $80-90 \%$ from a comparison of the peak areas. The recoveries were sufficiently good to match those of conventional glycoproteomic studies.

Ovalbumin consists of 385 amino acids with a sequence mass of $43 \mathrm{kDa}$, and has a single $\mathrm{N}$-glycosylation site at Asn ${ }^{105} .{ }^{20}$ This glycoprotein therefore contains 33 cleavable sites by trypsin digestion. Figure 5(a) presents a TIC chromatogram of the tryptic digests analyzed using LC/ESI-MS. Retentates as Con A complexes indicated appreciably simple TIC chromatograms (Fig. 5(b)). Furthermore, EIC indicated that glycopeptides bearing a common sequence, YNLTSVLMAMG TTDVFSSSANLSGISSAESLK, were eluted at $\approx 22.5 \mathrm{~min}$. These glycopeptides were difficult to resolve based on the difference in the oligosaccharide structures. The EIC spectra are portrayed in Fig. 5(c). We could not calculate the recoveries because of insufficient resolution of the glycopeptides. However, the detection of peak 4 corresponding to glycopeptides of oligosaccharide $\mathrm{E}$ suggested that the recovery of this method is quantitative because the content of oligosaccharide is less $1 \%$ of the total carbohydrate content, the binding constant to Con A is ca. $10^{3} \mathrm{M}^{-1}$.
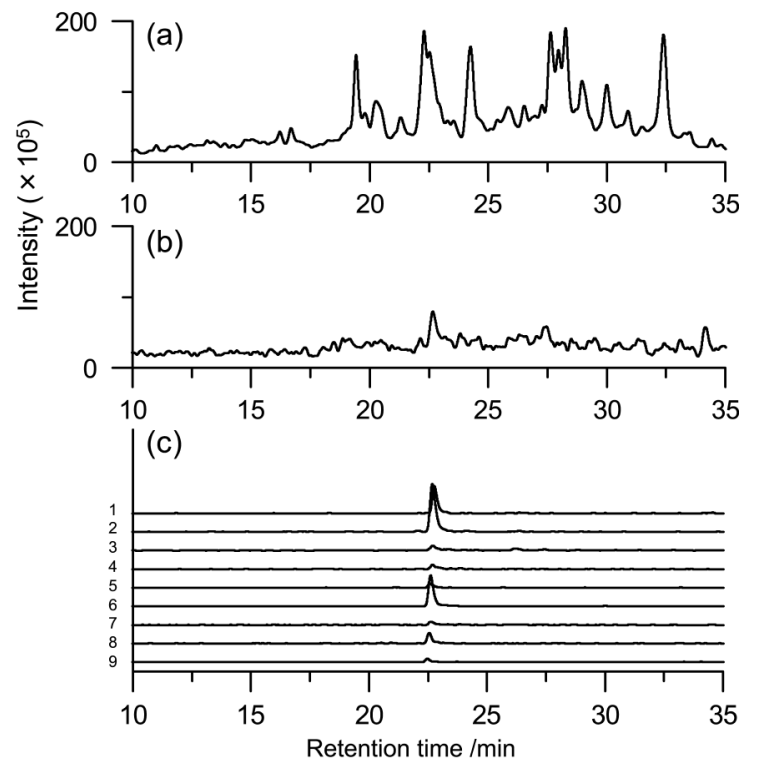

\begin{tabular}{ccccl}
\hline No. & $\begin{array}{c}\text { measured } \\
m / z\end{array}$ & $z$ & $\begin{array}{c}\text { glycosylation } \\
\text { site }\end{array}$ & \multicolumn{1}{c}{$\begin{array}{c}\text { glycan structure with identification } \\
\text { based on } m / z\end{array}$} \\
\hline 1 & 1542.34 & 3 & & {$[\mathrm{Hex}]_{5}[\mathrm{HexNAc}]_{2}$} \\
2 & 1596.34 & 3 & & {$[\mathrm{Hex}]_{6}[\mathrm{HexNAc}]_{2}$} \\
3 & 1610.20 & 3 & & {$[\mathrm{Hex}]_{5}[\mathrm{HexNAc}]_{3}$} \\
4 & 1625.68 & 3 & & {$[\mathrm{Hex}]_{4}[\mathrm{HexNAc}]_{4}$} \\
5 & 1650.35 & 3 & Asn $^{293}$ & {$[\mathrm{Hex}]_{7}[\mathrm{HexNAc}]_{2}$} \\
6 & 1677.68 & 3 & & {$[\mathrm{Hex}]_{5}[\mathrm{HexNAc}]_{4}$} \\
7 & 1691.35 & 3 & & {$[\mathrm{Hex}]_{4}[\mathrm{HexNAc}]_{5}$} \\
8 & 1745.35 & 3 & & {$[\mathrm{Hex}]_{5}[\mathrm{HexNAc}]_{5}$} \\
9 & 1799.35 & 3 & & {$[\mathrm{Hex}]_{6}[\mathrm{HexNAc}]_{5}$} \\
\hline
\end{tabular}

Fig. 5 Total ion current chromatograms of the reversed-phase LC/ESI-MS analysis of glycopeptides derived from ovalbumin digested with trypsin before (a) and after (b) specific extraction with Con A. Extracted ion chromatograms (c) correspond to trivalent molecular ions of glycopeptides with specific sequences listed in the table. Other conditions are identical to those for Fig. 4.

Human serum transferrin has two glycosylation sites at $\mathrm{Asn}^{432}$ and $\mathrm{Asn}^{630}$, which bears biantennary complex-type oligosaccharides with two $\alpha 2,6$-linked NeuAc. ${ }^{21}$ Therefore, $\alpha 2,6$-linked NeuAc recognizing SSA was chosen to trap transferrin-derived glycopeptides. Because of the higher molecular weight of transferrin (which contains 85 cleavable sites), many peaks were observed on TIC chromatograms (Fig. 6(a)). After extraction with SSA lectin, most peaks disappeared from the chromatogram; the peaks at 20,22.5, and 24.5 min remained. As shown in EICs, these corresponded to glycopeptides including $\mathrm{Asn}^{630}$ and $\mathrm{Asn}^{432}$, respectively. Moreover, we found minor components of $\mathrm{Asn}^{630}$ glycopeptides bearing a triantennary oligosaccharide and a fucosylated biantennary oligosaccharide, ${ }^{22}$ which was not observed in LC/MS analyses of the original tryptic digests of this glycoprotein. We also applied this method to other lectins, such as Maackia amurensis lectin (MAM) specific for $\alpha 2-3$-linked NeuAc. The extract indicated no peaks (data not shown), as expected from its specificity. Although some unidentified peaks were observed at 26-30 min on TIC chromatograms of the extract, this method appeared to be useful for the analyses of glycopeptides in tryptic digests of glycoproteins. 


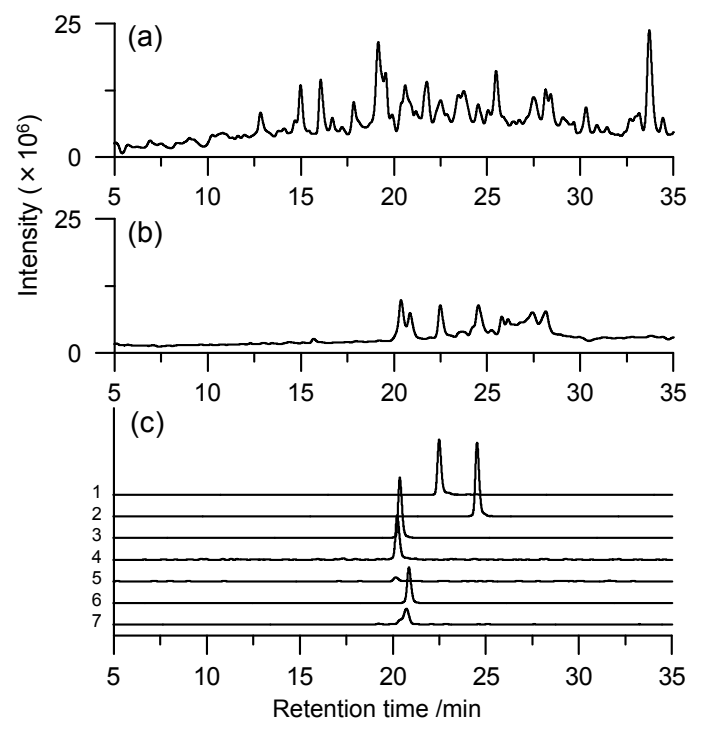

\begin{tabular}{|c|c|c|c|c|c|}
\hline No. & $\begin{array}{c}\text { measured } \\
m / z\end{array}$ & $z$ & $\begin{array}{l}\text { glycosylation } \\
\text { site }\end{array}$ & $\begin{array}{l}\text { glycan structure with identification } \\
\text { based on } m / z\end{array}$ & peptide $\bmod ^{* 1}$ \\
\hline 1 & 1227.18 & 3 & \multirow{2}{*}{$\operatorname{Asn}^{432}$} & {$[\mathrm{Hex}]_{5}[\mathrm{HexNAc}]_{4}[\mathrm{NeuAc}]_{2}$} & \multirow[b]{2}{*}{ PyroCMC ${ }^{* 2}$} \\
\hline 2 & 1222.55 & 3 & & {$[\mathrm{Hex}]_{5}[\mathrm{HexNAc}]_{4}[\mathrm{NeuAc}]_{2}$} & \\
\hline 3 & 1382.28 & 3 & \multirow{5}{*}{$\operatorname{Asn}^{630}$} & {$[\mathrm{Hex}]_{5}[\mathrm{HexNAc}]_{4}[\mathrm{NeuAc}]_{2}$} & \\
\hline 4 & 1430.92 & 3 & & {$[\mathrm{Hex}]_{5}[\mathrm{HexNAc}]_{4}[\mathrm{dHex}][\mathrm{NeuAc}]_{2}$} & \\
\hline 5 & 1503.99 & 3 & & {$[\mathrm{Hex}]_{6}[\mathrm{HexNAc}]_{5}[\mathrm{NeuAc}]_{2}$} & \\
\hline 6 & 1376.59 & 3 & & {$[\mathrm{Hex}]_{5}[\mathrm{HexNAc}]_{4}[\mathrm{NeuAc}]_{2}$} & PyroQ $^{* 3}$ \\
\hline 7 & 1430.92 & 3 & & {$[\mathrm{Hex}]_{5}[\mathrm{HexNAc}]_{4}[\mathrm{dHex}][\mathrm{NeuAc}]_{2}$} & PyroQ $^{* 3}$ \\
\hline${ }^{*} 1, \mathrm{n}$ & $\begin{array}{l}\text { fication } \\
\text { glutamic }\end{array}$ & & (1) & $\begin{array}{l}* 2, \text { deamination of alkylated cysteir } \\
\text { eptide }\end{array}$ & N-terminus; \\
\hline
\end{tabular}

Fig. 6 Total ion-current chromatograms of the reversed-phase LC/ESI-MS analysis of glycopeptides derived from human transferrin digested with trypsin before (a) and after (b) specific extraction with SSA. Extracted ion chromatograms (c) correspond to trivalent molecular ions of glycopeptides with specific sequences listed in the table.

\section{Conclusions}

Two methods were developed for the specific recovery and enrichment of glycoconjugates using free lectin solutions. The AP derivatives of ovalbumin oligosaccharides were fractionated based on their affinity for lectins. $\mathrm{RCA}_{120}$ enabled the fractionation of galacto-oligosaccharides from agalacto-oligosaccharides, and WGA enabled fractionation of bisected hybrid-type oligosaccharides from high mannose-type oligosaccharides. Complexation with Con $\mathrm{A}$ in the presence of methyl $\alpha$-mannoside also enabled the discrimination of oligosaccharides based on the number of mannose residues in their non-reducing end. Lectin complexation on an ultrafiltration membrane enabled the enrichment of glycopeptides for LC/MS analyses. In addition, Con A affinity was useful for the enrichment of glycopeptides derived from ribonuclease B and ovalbumin. Moreover, SSA enabled the enrichment of NeuAc-containing glycopeptides in tryptic transferrin. These methods yielded results that were comparable with those obtained using affinity chromatography with plant lectins.

\section{Acknowledgements}

This work was supported by the "High-Tech Research Center" Project for Private Universities: a matching fund subsidy from the Ministry of Education, Culture, Sports, Science and Technology (MEXT), Japan, 2007.

\section{References}

1. I. J. Goldstein, R. C. Hughes, M. Monsigny, T. Osawa, and N. Sharon, Nature, 1980, 285, 66.

2. O. Mäkelä, Ann. Med. Exp. Biol. Fenn., 1957, 26, 11.

3. N. Sharon, J. Biol. Chem., 2007, 282, 2753.

4. T. Mega, H. Oku, and S. Hase, J. Biochem. [Tokyo], 1992, $111,396$.

5. S. Honda, S. Suzuki, T. Nitta, and K. Kakehi, J. Chromatogr., 1988, 438, 73.

6. K. Nakajima, M. Kinoshita, Y. Oda, T. Masuko, M. Kaku, N. Shibuya, and K. Kakehi, Glycobiology, 2004, 14.

7. K. Nakajima, Y. Oda, M. Kinoshita, and K. Kakehi, J. Proteome Res., 2003, 2, 81.

8. J. Hirabayashi, J. Biochem. [Tokyo], 2008, 144, 139.

9. R. D. Cummings and S. Kornfeld, J. Biol. Chem., 1982, 257, 11235.

10. E. D. Green, R. M. Brodbeck, and J. U. Baenziger, J. Biol. Chem., 1987, 262, 12030.

11. M. Madera, Y. Mechref, and M. V. Novotny, Anal. Chem., 2005, 77, 4081.

12. L. F. Fraguas, J. Carlsson, and M. Lönnberg, J. Chromatogr., A, 2008, 1212, 82 .

13. A. Monzo, G. K. Bonn, and A. Guttman, Trends Anal. Chem., 2007, 26, 423.

14. Z. Yang and W. S. Hancock, J. Chromatogr., A, 2004, 1053, 79.

15. J. H. Hong, S. E. Duncan, S. F. O'Keefe, and A. M. Dietrich, Food Chem., 2009, 113, 180.

16. A. Kekwick and R. K. Cannan, Biochem. J., 1936, 30, 227.

17. S. Suzuki, K. Kakehi, and S. Honda, Anal. Biochem., 1992, 205, 227.

18. H. Nakagawa, Y. Kawamura, K. Kato, I. Shimada, Y. Arata, and N. Takahashi, Anal. Biochem., 1995, 226, 130.

19. T. H. Plummer and C. H. W. Hirs, J. Biol. Chem., 1964, 239, 2530.

20. R. D. Palmiter, J. Gagnon, and K. A. Walsh, Proc. Natl. Acad. Sci. U. S. A., 1978, 75, 94.

21. M. G. C. M. P. Team, Proc. Natl. Sci. U. S. A., 2002, 99, 16899.

22. Y. Satomi, Y. Shimonishi, T. Hase, and T. Takao, Rapid Commun. Mass Spectrom., 2004, 18, 2983. 